\title{
Conceptual Typography Between form Significance and Persuasion
}

\author{
Nermin El Sayed Ali Ahmed Abd El Halim
}

Lecturer, Higher Institute of Applied Arts - Advertising Section, Fifth Settlement, Egypt

\section{Keywords:}

conceptual typography, form, persuasion

\begin{abstract}
:
In the rapidly changing world of technology, sciences and the visual arts, there has begun a cultivating interest in the creative process as a fundamental human requirement, especially in the fields of art and design. When considering the field of conceptual typography, one must be made well aware that the letter, which is the basic component of the word, has become of two importances; which are preserving the readability of the written word and trying to achieve a communicative process that stimulates the receiver's senses. The written word thus transforms from being "simply" a visual form of communication, and a symbol indicating a certain object that conveys meaning, into an entity of its own, that delivers a certain message with a certain function that may arouse feelings and emotions in its receiver's psyche. This process is capable of achieving visual satisfaction due to the honesty and simplicity found in the structural composition of its formation, as it communicates with the receiver to the point of persuasion, motivation, and curiosity to participate in decoding the design and reading the meanings hidden within. The importance of studying conceptual typography's ability to persuade its audience is largely due to the scarcity of existing research concerned with the field in general and with their symbolic connotations in particular. The research focuses on answering the following question: To what extent could the field of conceptual typography stand to benefit from the symbolic and formal connotations of Arabic letters in achieving and increasing persuasion? And the research aims to achieve persuasion by taking advantage of the analysis of Arabic calligraphy and finding innovative symbolic design solutions that confirm the significance of the figure. To this end, the research follows the descriptive analytical methodology based on gathering information to build its theoretical framework and design a framework as a guidelines that helps the graphic designer in achieving the symbolic significance of typography and achieving persuasion by presenting some examples of works designed by the researcher.
\end{abstract}




\section{Introduction:}

The typography of Arabic calligraphy holds a prominent position in the field of graphic design due to the distinction of its design elements and their visual impact. As a design element, Arabic calligraphy is a quite malleable subject that can easily be formed and reformed through elongation, overlap, overlaying and integration with other design elements. It also bestows a lot of vitality to any design, regardless of its nature. Through analyzing Arabic letters and their linear formations, and in concentrating on their basic forms it slowly becomes obvious how their figures contain geometric shapes such as squares, triangles and circles.

Numerous studies have shown that Arabic letters possess a magnificent creative and symbolic energy that on one hand links memory to art and to cultural and linguistic identity on the other. It has also been found that these letters (symbols) may refer to more than one connotation, as they are varied and intertwined, and their meaning could very well differ from one society to another Symbols are employed to facilitate receivers' understanding and awareness - thus effective usage of the symbol leads to successfully delivering the targeted message audiences of various languages and nationalities. Symbols are also known to stimulate the receiver's mind, in enticing curiosity to participate in decoding designs and understanding the meanings they wish to convey. It is only in the success of the symbol in persuading its receiver that we achieve successful graphic design. (1)

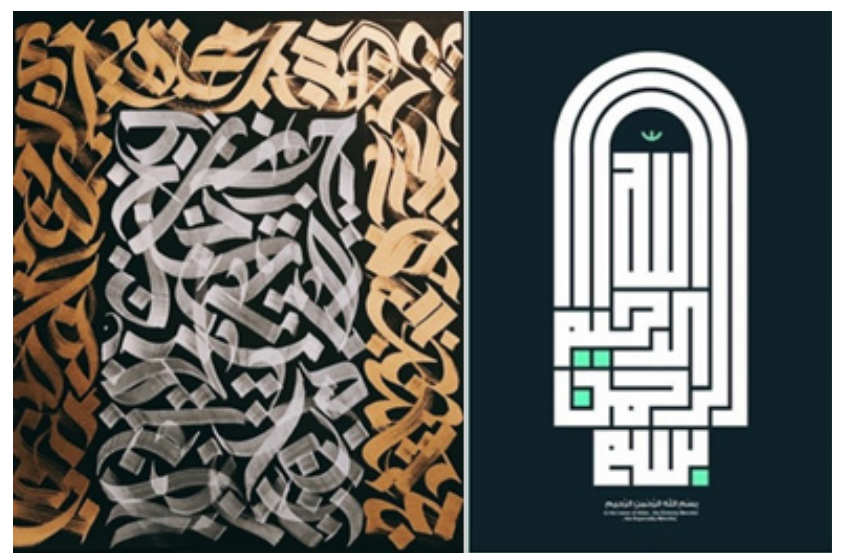

Figure (1)

shows the aesthetic beauty of Arabic calligraphy.

\section{Aesthetic Elements of Arabic Calligraphy in Conceptual typography}

Arabic calligraphy has undergone a lot of evolution since the olden cursive and ornamental patterns. In doing so, it aligned itself with the needs and functions of the times, and its artistry developed rapidly in turn. Interest was not cultivated in portraying the singular spelling of letters, but also moved to many other similarly traditional Arabic calligraphy styles, that were also developed to fit the needs of contemporary graphic design.

Graphic designers have also excelled in presenting designs that showcase contemporary aesthetic values, and have greatly expanded the scope of rejuvenating the field of calligraphy and design, to form a novel school of graphic design, characterized by creativity and clever employment of typographic design to suit inventive and developmental breakthrough from basic symbolism of calligraphy.

The aesthetic elements of Arabic calligraphy are closely related to advertisement designers' understanding of the nature of Arabic calligraphy, as its aesthetic value is achieved by employing those aesthetic elements. This is achieved by 'filling in' various types of Arabic fonts and shapes in artistic formations (composing squaring - scale and proportion - flexibility - simplicity - entanglement and overlap). (2)

\subsection{Composing}

It means creating and showcasing new proportions with different ratios and standards when connecting letters. There are certain fonts such as "alnaskh" and "al ta'aliq" and "al raqa'a that accept composing.

\section{2 .Squaring}

Is the ability to form geometrical shapes and angles, using letters. The square "kufi" font is considered the most appropriate in achieving this property, as its written depictions have displayed naturally geometric shapes such as squares, , triangles and rectangles. (3)

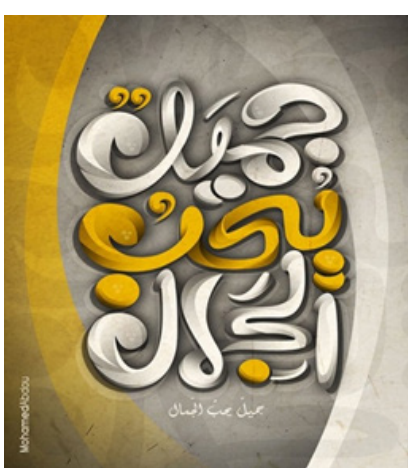

Figure (2) shows handwriting composition.

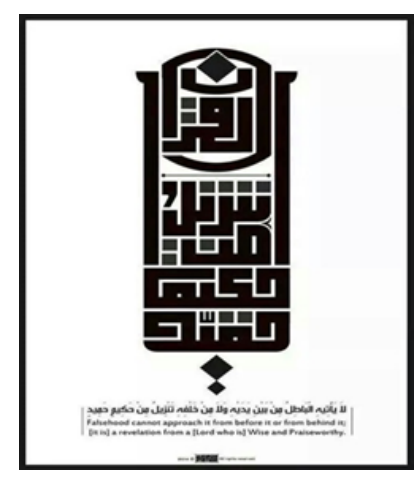

Figure (3) shows squaring in Arabic calligraphy. 


\section{3. scale and proportion}

Is a process of creating proportionality between the elements of Arabic calligraphy, from font, to point, to enunciations and design.

\section{4. flexibility}

Is considered to be one of the most important aesthetic elements in Arabic calligraphy, as it makes best use of the calligraphy's susceptibility to various aesthetic formations like curvature and rotation, and their ability to stretch and elongate in satisfactory ways to achieve the aesthetic values of design.

\subsection{Simplicity}

It means the visualization of letters and words, free of any affectations or embellishments in shapes or combinations.

\section{Fine Standards for the Use of Letters and Writ- ing in Conceptual typography}

\subsection{Structuring the Connected Sentence}

It is the process of installing, joining and assembling signs and letters, then rounding and extending them to emulate geometric forms such as squares, circles, or rectangles to form words and sentences in a typographical fashion. This is done so that they would merge into a holistic form by employing the typographic space aesthetically to achieve parity and consistency in forming a typographic symbol. (4)

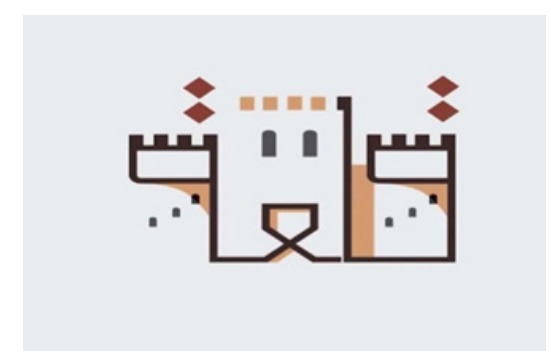

Figure(4) shows structuring the connected

\subsection{Simplicity and Clarity of Letters}

It is the process of constructing, joining, and grouping signs, letters, and numbers to form words and sentences in a conceptual typography fashion, so that all of them merge in a holistic form by making use of the aesthetic typographic space.

\subsection{Readability}

The height and thickness of the letter must be taken into account, while also considering the ratio between the height of the letter and the height of the design at hand. The degree of clarity of letters varies according to the advertising medium and the intended target of the symbol, as in Figure (5) which shows how the designer depended on the symbolic meaning of Fri- day and linked it to the form of the minaret. There is, however, an observed lack of clarity in the first and third letters in the word Friday, which affect the ease of reading.

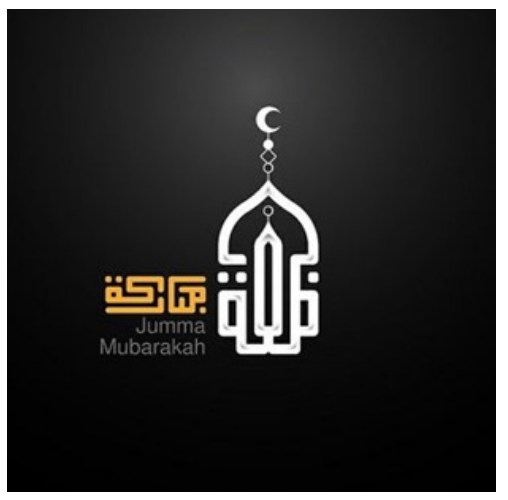

Figure (5) shows Readability

\section{2 .4 . Space}

It is the distance between letters, and it is the result of the visual arrangement of the letters' appearance inside the typographical composition, whether in convergence or in distance. Figure (6) shows how the designer exploits the typographic space to emphasize the significance of form and meaning, while using distinctive texture as well.

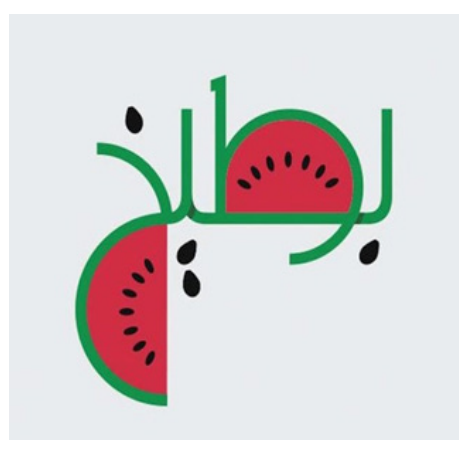

Figure (6) shows Typographic space.

\subsection{Visual Arrangement}

It is the arrangement of elements in a chain fashion, ranging from the most dominant to the least dominant in the conceptual typography. The more varied the characteristics of the letters are, in visual sequence, the more possible it is to determine which of these letters is in the dominant position and which falls next.

\subsection{Organization within a conceptual Typography Design}

Signifies organizing letters and words so that spaces exist between them, so that they do not blend when looking at them. There are 3 directions to follow when using letters in a design intended to persuade an audience: 
- The shape of letters from an aesthetic view point.

- The arrangement of letters and script within the design.

- Suitability for the required cause. (4)

\section{The Conceptual typography of Arabic calligraphy} Conceptual typography is designing using Arabic letters, calligraphy, distribution and arrangement in order to obtain an innovative work that has a symbolic meaning, which includes ornamental and symbolic aesthetics in letters' writings to achieve beyond simple visual effects. (5)

Arabic calligraphy is a form of handwriting that carries sound values. However, its formulation also adds intellectual, cultural, moral and philosophical value to its sounds.

If we take into consideration the letter (I) as an expression of a certain sound, standing vertically erect, without the assistance of other letters - this provides it with emotional properties that belong to our culture, as if it is the mainstay that helps in building all other letters, as it represents the concept of stability, support and beginning.

We also observe other letters, such as (و ،ف ،ع ،ض ،ص (2) $\tau)$, that depend on bending to exist. Bending expresses flexibility and containment, and when we contemplate the Arabic alphabet, we find that it provides wonderful space in these spaces for communication.

Almost all letters, such as (0- $-0-\tau-\jmath-9)$, leave us a vacuum, whether closed or open, and whether a single or multiple space, allowing for a sense of diversity as well.

The other characteristic of the Arabic alphabet is its natural tendency during writing for curvature, which alludes to that of trees and rivers, which gives a sense of freedom in infinite space, and the ability to adapt and harmonize with the other, and avoid conflict. All the above mentioned is what Arabic calligraphy achieves in a truly miraculous way, and this is exactly what we do not find in the Latin script, for example, where its acute angles represents a basic dimension that predicts completely different values, such as conflict, clashing, frigidity and interruption. (6)

\subsection{Psychological Implications of Conceptual ty- pography}

-Horizontal writings: are considered a rule that works above all and gives a sense of breadth. --Horizontal writings suggest stability, stillness and steadiness.

-Vertical writings: give a sense of strength, sophistication, and supremacy.

-Italic writings: give a sense of imbalance, which en- riches the feeling of movement and dynamism. -Free Writings: Flexible or curved writings suggest flexibility, vitality, and a natural flow.

Words represent the link between visual forms and understanding, so it is necessary to study them in detail, as they represent an integral part of all elements of visual communication, whether written or illustrated. Figure (7) shows the designer using the shape of the eagle and depicting it through the letters of the word in a way that strongly suggests the power of the eagle.

(7)

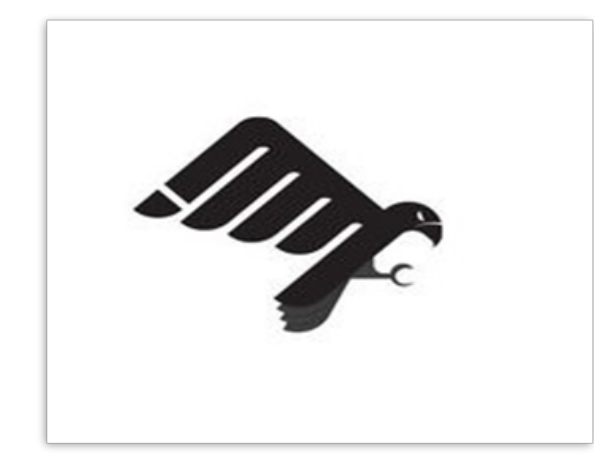

Figure (7) shows

Psychological Implications of Conceptual typography

\subsection{Indications of Form in Conceptual typography}

Conceptual typography depends on the letters or Arabic fonts, distributing and arranging them to obtain an innovative creative work that carries a symbolic meaning, either by using the word or word and form together to communicate the significance of the form and its meaning. The character in the design has an effective role in confirming

the meaning and significance of the expressive form of the message and convincing the receiver of the idea behind the typographic symbol.

As writing helps in moving the visual and psychological factors of the receiver of the typographic design as a whole, there are also some designs that are entirely built using the personality of a particular font, as in Figure (8), in which the designer used the character of the letter to express the shape of the owl through the shape of the letters forming the word and spaces. The typographical elements found in the design play an important role in confirming the character's capabilities to express through properties such as general atmosphere, feelings and stimulated impressions. Therefore, there are different variations of the patterns of different lines in thickness, softness, straightness and such. (8) 


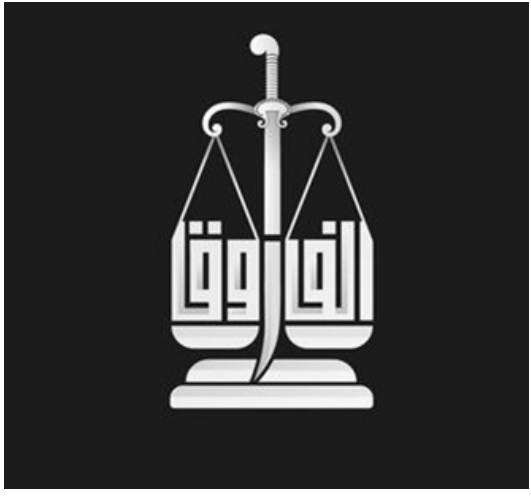

Figure (8) shows

the relationship between shape and typographical elements.

Shapes have a special significance in influencing the receiver, and knowledge of these effects is important in designing conceptual typography, and in the successful use of the indications of shapes in the design of the symbol.

Typography leads to arousing different emotions and motives within the receiver towards the subject and content of the component form of the design of the conceptual typography. The designer should always be concerned with the psychological aspects within the receiver with the same level of interest in the artistic and aesthetic aspects.

The language of the form is considered one of the most important visual means of communicating information, as it is the medium or function. It has a specific meaning, and its indications play a major and important role. (9)

The formal indications carry certain pieces of information and messages conveyed to the receiver, so the form conveys the customs and social traditions that are among the pillars of communication between humans, as in Figure (9), which clarifies the word Al-Faruq, the distinguished title of Omar Ibn Al-Khattab where the letters of the word were formed in the form of scales that symbolize the justice that Omar

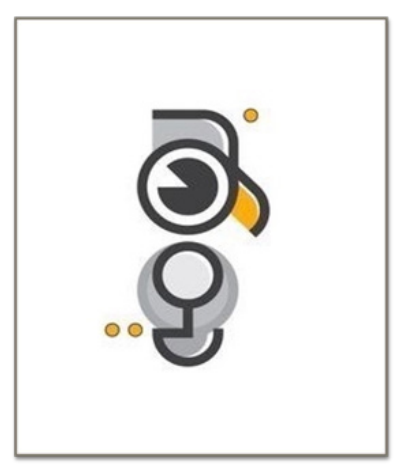

Figure (9) shows the semantic meaning of shapes in typography.
Ibn Al-Khattab was famous for.

\subsection{Overtones of Basic Geometric Figures 3.3.1 Stability in Shapes}

The pyramid is one of the forms associated with stability and is considered the most stable form in the case of placing one of its pillars centered on the surface of the earth, but if it focuses its head on the surface of the earth it gives an indication of imbalance and it appears to those who see it as if it will collapse or that it will fall until it regains the status of stability on one of its pillars.

\section{.3.2 Simplicity in Shapes}

The square shape is a symbol of simplicity and specific proportions and is a static form that does not have a distinct direction-finding feature. All shapes of the four sides are a product of the origin of the square shape with the different measurement of its sides and angles. Much like the triangle, the square provides a sense of stability if it rests on one of its sides, but if it is based on one of its corners, it becomes a moving shape that suggests instability.

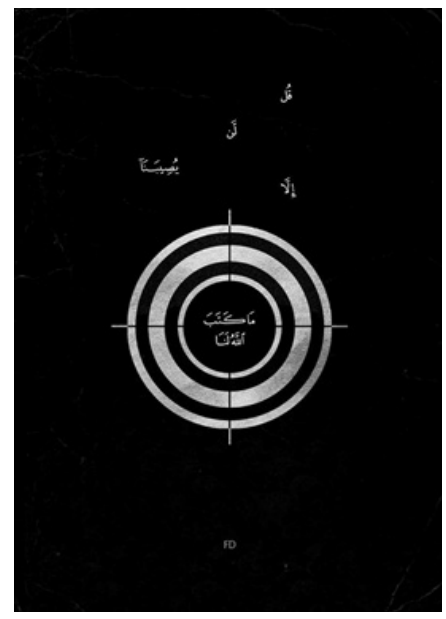

Figure (10) shows overtones of geometric shapes.

\subsubsection{Centralization in Shapes}

The circle with a stable shape is also considered a center of its surroundings. When a circle is placed somewhere it creates a central sense of focus. The movement of the circle around its axis gives different forms of the ellipse as in Figure (10), which shows the symbolic use of the shape of the circle in confirming the symbolic significance of the Quranic verse "Nothing will happen to us except what Allah has decreed 
for us". By using the shape of the circle in the center of the design, in a manner similar to target practice, with the verses "Allah has decreed for us" placed in the center of the target to emphasize the imperative of its occurrence while the rest of the verse is scattered outside shooting range, to ensure that it does not happen. (10)

\section{Conceptual typography and Persuasion}

Persuasion is an intellectual and formal process in which one of the parties tries to influence the other and convince him of a specific idea or approach with specific effects. (11) It can also be considered as a process aimed at changing the positioning or behavior of a person or group towards a specific event. This is done using written or spoken words to convey a message. Persuasion is a deliberate attempt on the part of the sender to change the receiver's beliefs, directions, or behaviors by conveying certain messages. (12) The importance of persuasion when it comes to conceptual typography lies in the necessity of realizing that it is not simply a written text, but rather a form that carries within it a message with a meaning and function that may entice the feelings and emotions of the receiver, and guarantee their mental and psychological satisfaction, due to the sincerity and simplicity found in its structural composition. To this end, the receiver's mind is motivated by curiosity to participate in decoding the design as well as reading within its meanings.

\subsection{Meaning-Creation Strategy}

Conceptual typography depends on the strategy of creating meanings to persuade the receiver with the message addressed to him. This strategy does not only address what is written or spoken. Symbols, signs and shapes also have meanings, and they are also considered persuasive expressions in the form of allusions and insinuations found within the design of the typographic symbol, as in Figure (11) which clarifies the persuasion strategy to build meaning.

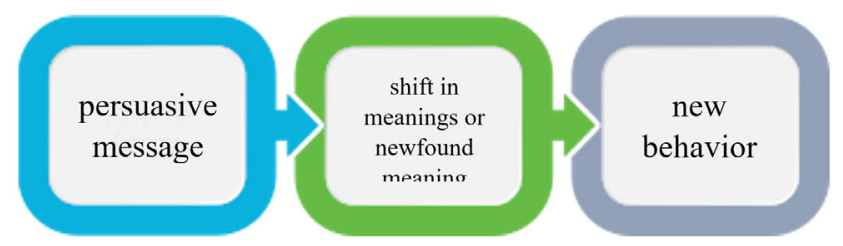

Figure (11) shows the Meaning-Creation Strategy
This takes place throughout three stages; which are the persuasive message contained in the typographic symbol and its connotations that lead to newfound meanings or a shift in meaning-making, which leads to a behavioral or emotional reaction within the receiver as a result of its influence Persuasion is a process that is not limited to receiving information, but includes understanding all symbols, insinuations and signs, and not passively receiving them in a superficial manner. This largely depends on persuasion factors and the ability to communicate semantic meaning of the form at hand. Therefore, it is necessary to integrate the elements of conceptual typography (symbols, suggestions, insinuations + what is written and what is spoken) to lead to a contribution to the persuasive process translated into conceptual typography.

\subsection{Rules and Principles to Abide by During the Persuasive Process}

There are a set of rules and principles that must be in place in order to reach the goal of persuasion:

- The persuader must be convinced with the idea in order to persuade the receiver.

- The clarity, integrity and reasonableness of the idea, and the clarity of the significance of the form and the content of the message expressed by the symbol's typography.

- The persuader's ability to clarify and explain the idea.

- Application of the fundamental criteria for usage of letters and scripts in conceptual typography (14).

\section{The Researcher's Analytical Framework}

The researcher, through collecting and analyzing information, and building the theoretical framework, came to find that methods of design and work as a guideline for the graphic designer, and contribute to the actual design process of typographic symbols, which works to confirm the significance of the form to achieve persuasion, as shown in Figure (12). Based on the elements of Arabic calligraphy, visual space, and the employment of symbols and shapes, the target is to ensure that the receiver participates to reach the signifier in an innovative way that works to attract the receiver's attention. To achieve visual persuasion, the researcher presents below some examples of her work. 


\section{Design Methods for Achieving Shape in conceptual Typography}

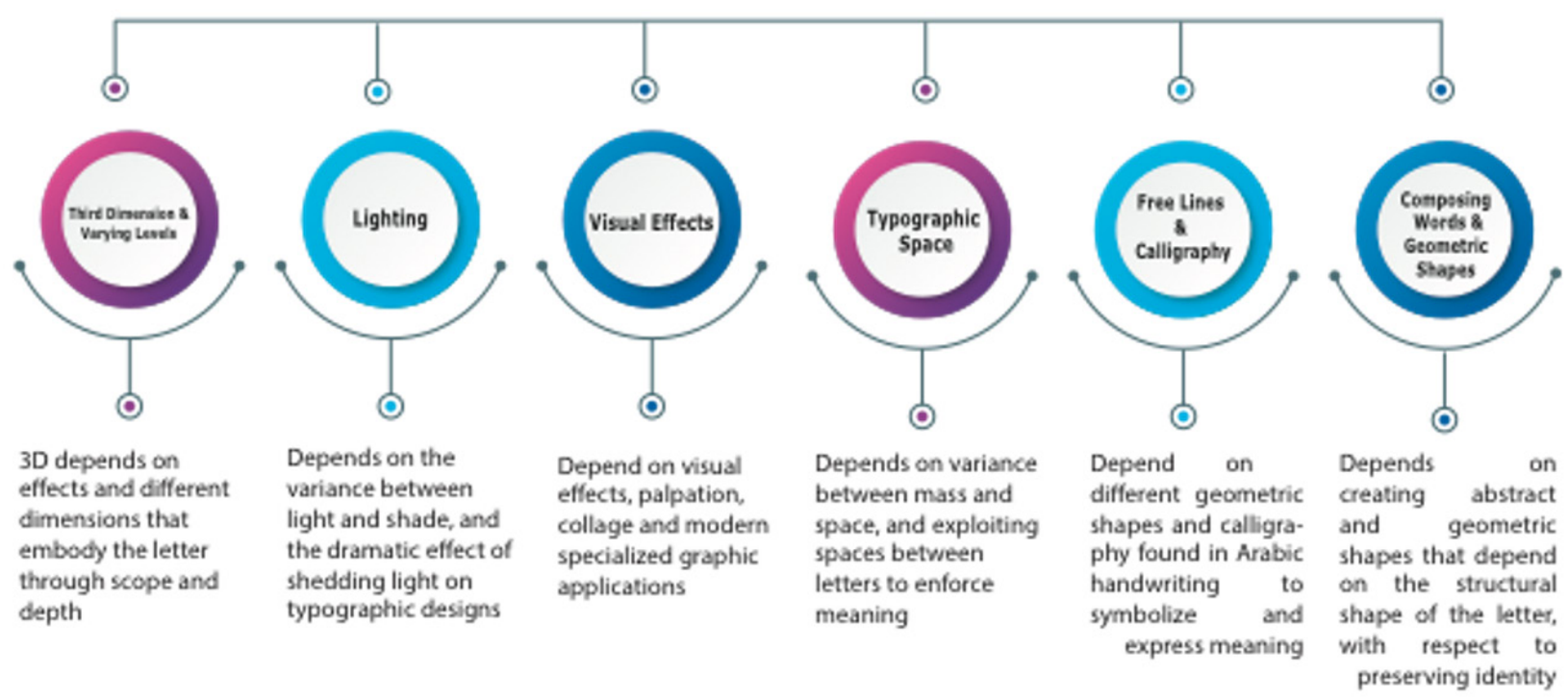

Figure (12) shows Design Methods for Achieving Shape in conceptual Typography

\subsection{Model (1): A style based on geometric shapes:}

The word ( تصميم ):

This experiment, in Figure (13), depended on the geometric shapes and the structural dimensions of the typography of Arabic calligraphy, by stripping the letters of the word design for geometric shapes, and expressing the elements of graphic design, embodying their linguistic meaning, and achieving the significance of the shape through the implementation of design elements in a way that preserves the readability of the word and achieves balance, unity and rhythm through chromatic repetition and maintaining the proportions of aesthetic letters.
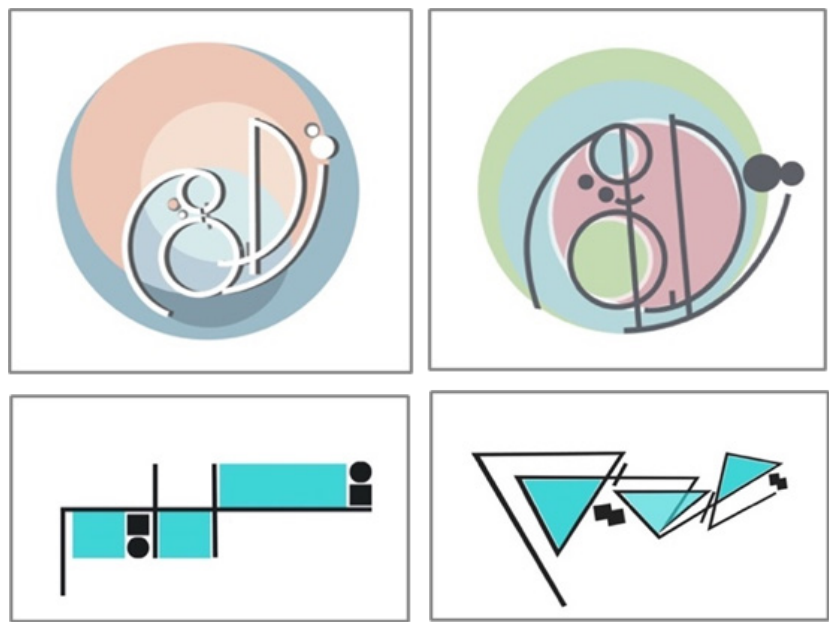

Figure (13) shows Model (1): A style based on geometric shapes

\subsection{Model (2): a style based on free line and callig- raphy:}

The word (كراكيب):

This experiment, in Figure (14), relied on the free line and the movement of straight lines intertwining in different directions, forming a word as syntax, to express the semantic and linguistic meaning of the word, and to confirm it visually through creating random and irregular interference between the lines, forming the word that appears through the contrast of thickness and color, forming them in lines scattered around in all directions with chaotic disorder.

The word (امي) :

This experiment, in Figure 14, was based on the free font and curved lines of the letters of the word 'mom', creating a heart shape. The researcher used curved lines to indicate softness and containment, as well as the red color of loving feelings associated with motherhood
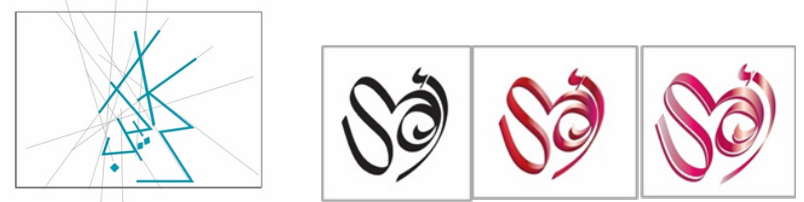

Figure (14) shows Model (2):

A style based on free line and calligraphy 


\subsection{Model (3): a style based on the typographic} space:

The word ( فراغ ):

In Figure (15), this experiment depended on the embodiment of the word 'space' by exaggerating the vacuum surrounding the word inside the square.

he word ( كis):

This experiment, in Figure (15), depended on the embodiment of the word 'mass' by exaggerating the size of letters while reducing the size of the topographic space between the letters to confirm the concept of the mass and its ratio to space.
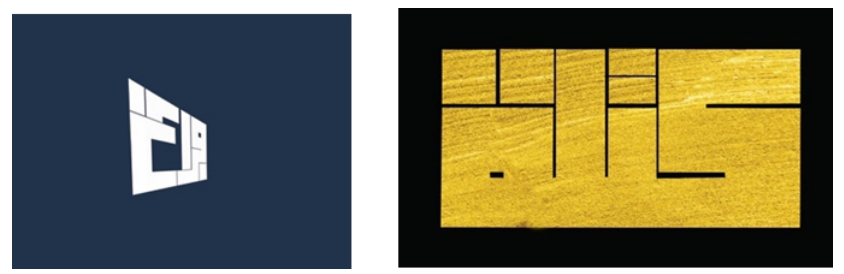

Figure (15) shows Model (3): A style based on typography space

\subsection{Model (4) a style based on visual effects:}

The word (تشويش ): Figure (16)

This experiment was based on the embodiment of the word's semantic meaning of it by using the glitch effect to give the illusion of vibration and distortion, along with clashing the direction of the letters, and confirming it with color

The word ( انفجار): as figure 16

This experiment was based on the embodiment of the semantic meaning of explosion by using the brush tool to give the effect of fragmentation of the letter particles as a result of exploding
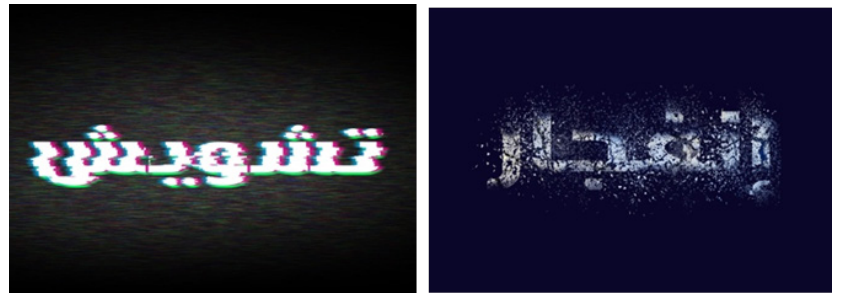

Figure (16) shows Model (4): A style based on visual effects

\subsection{Model (5) light-dependent style:}

The word (غموض): Figure (17)

This experiment was based on the embodiment of the semantic meaning of it through the influence of light, which clearly shows some of its parts, while hiding some other parts to indicate the meaning of the word 'ambiguity' The word (نور ): Figure (17)

This experiment depended on the semantic meaning of the word "light" through the influence of light, which clearly shows some of its parts while casting some other parts in shadow
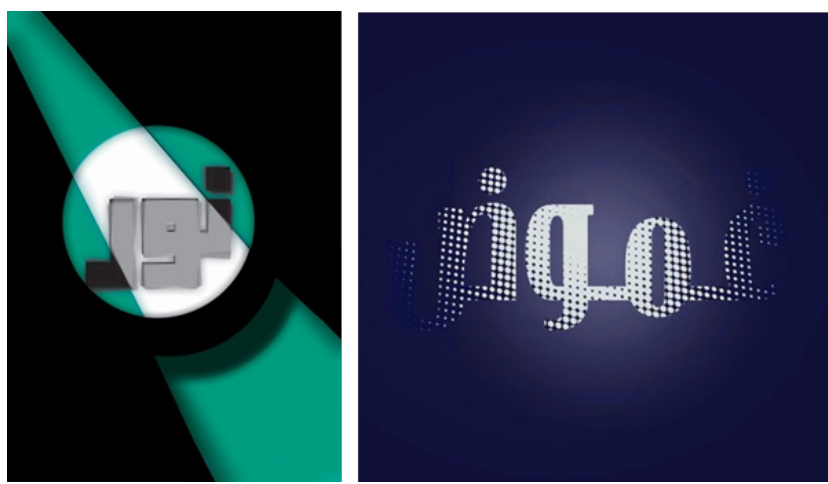

Figure (17) shows Model (5): A style based on lighting effect

\subsection{Model (6) a style depending on varying levels:}

The word ( تدرج): Figure (18)

This experiment was based on the embodiment of the semantic meaning of the word itself through the composition of the letters of the word in the form of steps on a staircase.

The word ( انكسار ): Figure (18)

This experiment was based on the embodiment of the semantic meaning of the word itself through the separating of parts of the word into two levels to convey shattering
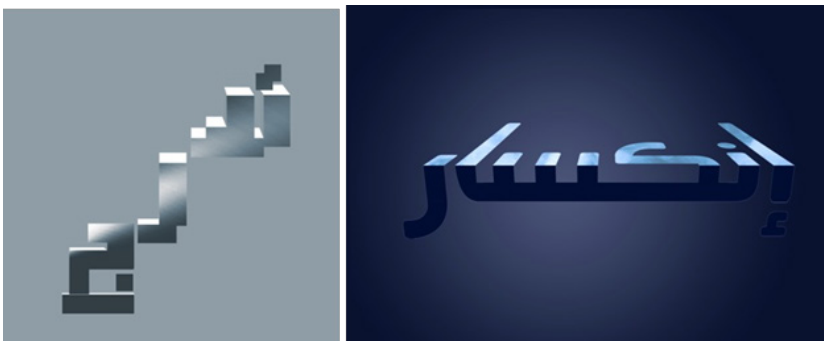

Figure (18) shows Model (6: A style based on varying levels 


\section{Results:}

- Dependence on the formal and symbolic dimension of the typography of Arabic calligraphy helps to enrich innovation, and has a large impact on the clarity of the idea and convincing the receiver of the content of the message.

- Formative connotations carry specific information and messages of deep innovative formulation that helps stimulate the mind and attention of the receiver.

- The alphabetic letter plays an effective role in confirming the meaning and significance of the form expressed in the message in designing typographic symbols and persuading the receiver.

- Knowing the connotations of shapes and their successful use in designing typographic symbols leads to arousing different emotions and motives of the receiver and convincing him of any certain idea.

- Persuasion is a process that is not limited to receiving information, but includes understanding all symbols, insinuations and signs and not receiving them on a superficial basis only. This matter depends on the methods of the persuading person and their ability to communicate the semantic meaning of the form translated into typographic symbols.

- The symbols used in the design of the typographic symbols vary between linguistic symbols of the Arabic language and non-linguistic characters.

- The significance of the shape in typographic symbols can be achieved and confirmed by the graphic designer following the following design methods: - Geometric shapes and the composition of the shape or image with the word through the structural analysis of the Arabic letter.

- Free calligraphy, enunciations, relying on the forms and fonts of Arabic calligraphy, and its letters' ability to extend and be flexible.

- Visual effects through the use of contacts, collage and modern techniques for the rigor of specialized design.

- The topographic space through the contrast between mass and space formed between letters

- Lighting and dramatic proportions resulting from the contrast between shadow and light.

- The third dimension and varying levels.

\section{Recommendations}

Recommendations for the typographer of Arabic calligraphy:

The typographer of Arabic calligraphy should consider:

- Investing in the global cultural repository as a re- newable source for modern and unique signs and symbols. To elaborate, the symbols used in design should have common meanings between different cultures.

- Paying attention to the graphic treatments of the typography of Arabic calligraphy, to confirm and support its significance in conceptual typography in addition to its linguistic significance.

- To have a high level of knowledge of the various indicators and the visual language of the receiver in a manner consistent with the nature and culture of their society.

- Recommendations for academic and technical institutes dealing with the field of teaching Arabic calligraphy

- Directing attention to increasing studies concerned with Arabic calligraphy.

- Increasing specialized studies on conceptual typography and symbolic dimensions of Arabic fonts and shapes.

\section{References:}

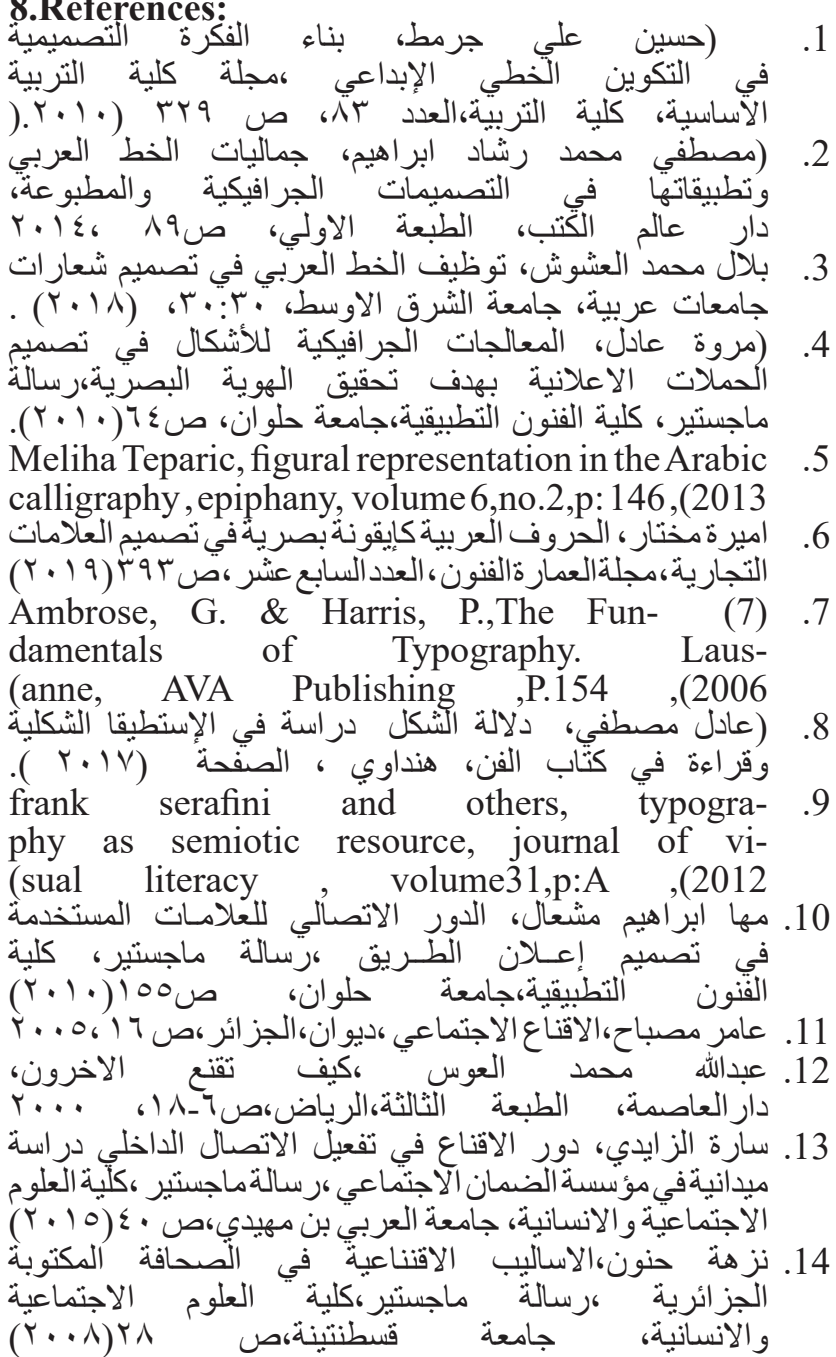

